\title{
Atual situação da pandemia no Brasil: inovação tecnológica, avanços na ciência e desafios
}

\author{
Current situation of the pandemic in Brazil: technological innovation, advances in Science and
} challenges

Situación actual de la pandemia em Brasil: innovación tecnológica, avances em la ciência y desafios

Thaís Paula de Araújo ORCID: https://orcid.org/0000-0001-8672-5325 Universidade Federal de São João del-Rei, Brasil E-mail: thaisp_araujo@ outlook.com

Luana Cristina Diniz Santos ORCID: https://orcid.org/0000-0001-8399-575X Universidade Federal de São João del-Rei, Brasil E-mail: luanadiniz.santos@gmail.com

Wanderson Duarte Penido ORCID: https://orcid.org/0000-0002-3688-6347 Universidade Federal de São João del-Rei, Brasil E-mail: wandersondpenido@gmail.com Gabriel de Menezes Yazbeck ORCID: https://orcid.org/0000-0003-1729-348X Universidade Federal de São João del-Rei, Brasil E-mail: gabriel@ufsj.edu.br

\begin{abstract}
Resumo
A pandemia da doença do Coronavírus de 2019 evidenciou que o mundo não estava suficientemente preparado para o enfrentamento de epidemias emergentes, expondo grande vulnerabilidade sanitária e socioeconômico global, principalmente em países subdesenvolvidos como o Brasil, sendo o segundo país com maior número de índices de contágio e óbitos, ultrapassando a marca de 600 mil óbitos. O objetivo dessa revisão é identificar fármacos e vacinas em desenvolvimento e discutir sobre os principais desafios da pandemia no Brasil. Com o rápido avanço da pandemia, iniciou-se uma corrida tecnológica para desenvolvimento de vacinas contra o coronavírus. Atualmente há mais de 300 vacinas em desenvolvimento contra o COVID-19, dentre elas 18 estão sendo desenvolvidas por institutos brasileiros. Além disso, foi aprovado o uso emergencial de 4 vacinas candidatas, são elas: Covisheield, Comirnaty, Coronavac e Janssen. $\mathrm{O}$ combate à pandemia no Brasil esbarra em obstáculos como falta de saneamento básico, limitado acesso aos serviços de saúde e à educação de qualidade, além de ações equivocadas ou omissões do poder público e da ampla difusão de desinformação sobre a doença, o que contribuiu para o aumento do número de casos, e esterçamento do sistema de saúde. Atualmente, o avanço da vacinação 58,25\% da população adulta com esquema vacinal completo tem implicado na queda consistente de casos e óbitos, graças a uma sólida estrutura estatal de programa de vacinação de alta capilaridade e alta aceitação popular à vacina.
\end{abstract}

Palavras-chave: Brasil; Ciência; Coronavírus; Inovação tecnológica.

\begin{abstract}
The 2019 Coronavirus disease pandemic showed that the world was not sufficiently prepared to face emerging epidemics, exposing great global sanitary and socioeconomic vulnerability, especially in underdeveloped countries like Brazil, being the second country with the highest number of contagion rates and deaths, surpassing the 600 thousand deaths mark. The objective of this review is to identify drugs and vaccines in development and discuss the main challenges of the pandemic in Brazil. With the rapid advance of the pandemic, a technological race for the development of vaccines against the coronavirus began. There are currently more than 300 vaccines under development against COVID-19, among them 18 are being developed by Brazilian institutes. In addition, the emergency use of 4 candidate vaccines was approved, namely: Covisheield, Comirnaty, Coronavac and Janssen. The fight against the pandemic in Brazil comes up against obstacles such as lack of basic sanitation, limited access to health services and quality education, in addition to mistaken actions or omissions by the public authorities and the widespread dissemination of misinformation about the disease, which contributed to the an increase in the number of cases, and a weakening of the health system. Currently, the advance in vaccination of $58.25 \%$ of the adult population with a complete vaccination schedule has resulted in a consistent drop in cases and deaths, thanks to a solid state structure of a vaccination program with high capillarity and high popular acceptance of the vaccine.
\end{abstract}

Keywords: Brazil; Science; Coronavirus; Tecnologic innovation. 


\begin{abstract}
Resumen
La pandemia de la enfermedad del Coronavirus 2019 mostró que el mundo no estaba lo suficientemente preparado para enfrentar epidemias emergentes, exponiendo una gran vulnerabilidad sanitaria y socioeconómica global, especialmente en países subdesarrollados como Brasil, siendo el segundo país con mayor número de tasas de contagio y muertes, superando las 600 mil muertos marca. El objetivo de esta revisión es identificar medicamentos y vacunas en desarrollo y discutir los principales desafíos de la pandemia en Brasil. Con el rápido avance de la pandemia se inició una carrera tecnológica para el desarrollo de vacunas contra el coronavirus. Actualmente hay más de 300 vacunas en desarrollo contra COVID-19, entre ellas 18 están siendo desarrolladas por institutos brasileños. Además, se aprobó el uso de emergencia de 4 vacunas candidatas, a saber: Covisheield, Comirnaty, Coronavac y Janssen. La lucha contra la pandemia en Brasil tropieza con obstáculos como la falta de saneamiento básico, el acceso limitado a servicios de salud y educación de calidad, además de acciones u omisiones equivocadas por parte de las autoridades públicas y la difusión generalizada de desinformación sobre la enfermedad, que contribuyeron al aumento del número de casos y al debilitamiento del sistema de salud. Actualmente, el avance en la vacunación del 58,25\% de la población adulta con un esquema de vacunación completo ha resultado en una caída constante de casos y muertes, gracias a una estructura de estado sólido de un programa de vacunación con alta capilaridad y alta aceptación popular de la vacuna.

Palabras clave: Brasil; Ciencias; Coronavirus; Innovación tecnológica.
\end{abstract}

\title{
1. Introdução
}

A síndrome respiratória aguda grave causada pelo novo coronavírus (SARS-CoV2), um vírus emergente patogênico, representa uma séria ameaça à saúde pública, sendo responsável pela recente pandemia mundial da doença do coronavírus de 2019 (COVID-19) (Zhang et al., 2020). Como um agente infeccioso das vias aéreas, transmitido pelo ar, a manifestação da doença pode apresentar sintomas como fadiga e fraqueza muscular, dispneia, dor no peito, dor de cabeça e espirros, redução de olfato e paladar, distúrbios cognitivos, artralgia e declínio na qualidade de vida, podendo afetar o sistema nervoso central, e os sistemas cardiovascular, renal, hematológico e gastrointestinal (Bliddal et al., 2021; Blomberg et al., 2021; Nalbandian et al., 2021).

A Organização Mundial da Saúde (OMS) declarou no dia 30 de janeiro de 2020 o surto de Coronavírus que se tornou uma emergência de saúde pública de interesse internacional, sendo elevada a classificação para pandemia em 11 de março de 2020. O número de infectados pela pandemia do COVID-19 é 248.467.363 casos confirmados e 5.027.183 óbitos global registrados até o momento (OMS, 2021). Dentre os países com maior número de infectados, destacamos o Brasil, que possui mais de 21 milhões de casos acumulados e cerca de 609 mil óbitos, sendo considerado o segundo país com o maior número de casos e com uma margem de número de mortes diárias que chegou à 4.249 (CONASS, 2021).

Desde o início da crise sanitária, esforços consideráveis têm sido colocados no desenvolvimento de fármacos e vacinas eficazes e seguras contra o SARS-CoV2 (Funk et al., 2020). Para garantir a segurança e eficácia de uma vacina é necessária uma série de protocolos que são divididos em 5 fases. (Brasil, 2020d). A pesquisa e desenvolvimento de vacinas podem normalmente ultrapassar 10 anos, no entanto, devido à pandemia causada de COVID-19, a OMS adotou um sistema “fast-track" para uso emergencial. Até o momento, cerca de 331 vacinas contra COVID-19 estão em desenvolvimento, dentre elas, 18 estão sendo desenvolvidas no Brasil com tecnologias nacionais (Brasil 2021).

Os desafios tecnológicos no combate à pandemia envolvem a busca por novo uso de medicamentos já existentes (Brito et al., 2020), e a busca de inovações na pesquisa, incluindo o desenvolvimento, avaliação e uso de novas terapias e vacinas, prioritárias nessa luta (Lima et al., 2021). Como incentivo no desenvolvimento de medicamentos inovadores decorrentes do setor farmacológico, a proteção de patentes pelo direito de Propriedade Intelectual (PI), pode ser fornecida como um incentivo à inovação no ecossistema econômico atual, assegurando aos titulares da invenção retorno pelo seus investimentos, na sua exploração econômica exclusiva, por determinado tempo e seguindo determinado critérios normativos (Van Overwalle, 2020; Zambrano, 2020).

No entanto, esse sistema de patentes pode envolver questões éticas de direitos de propriedade intelectual, como o licenciamento compulsório, devendo ser gerenciados com cautela, e deve ser deixado de lado considerações financeiras em 
favor de preocupações éticas ou morais, a fim de não dificultar o acesso aos fármacos e vacinas, especialmente quando se trata de enfrentar emergências globais de saúde, como a pandemia da COVID-19 (Bonadio \& Baldini, 2020).

Os desafios enfrentados pela pandemia são ainda maiores no Brasil, por fatores, como vasta extensão territorial, opções políticas questionáveis na esfera federal (Hallal, 2021) e grande desigualdade social (Werneck \& Carvalho, 2020), onde alguns estados o acesso ao sistema de saúde pode ser restrito e apresentarem dificuldades em realização de testes, rastreamento e de disponibilidade de profissionais, aumentando sua vulnerabilidade ao vírus (Moreira et al., 2020). Esta revisão tem, portanto, objetivo de fornecer uma visão geral da inovação tecnológica de fármacos e vacinas, no contexto da COVID-19, com destaque para a atuação da ciência frente à pandemia, além de realizar um estudo de caso do Brasil, apontando os principais desafios enfrentados pelos brasileiros frente à pandemia.

\section{Metodologia}

O presente estudo trata de uma revisão bibliográfica acerca da pandemia causada pelo coronavírus no mundo, com ênfase no Brasil, foram selecionados estudos com foco na inovação tecnológica, como o desenvolvimento de fármacos e vacinas, avanços na ciência e os principais desafios enfrentados no Brasil frente a pandemia. Abrangemos principalmente banco de artigos científicos como Google acadêmico, portal periódicos Capes, Scielo, Pubmed, mas também usamos dados públicos disponíveis em sites do governo do Brasil, e mundiais. Foram selecionados trabalhos publicados em 2020 e 2021.

\section{Pesquisa e desenvolvimento de fármacos e vacinas}

\subsection{Inovação Tecnológica ao contexto da pandemia}

A atuação da ciência frente a pandemia do coronavírus, em especial no setor farmacológico, está interligada com questões de Inovação Tecnológica e Propriedade Intelectual. Os medicamentos inovadores decorrentes do setor farmacológico que se caracterizem como novidade, atividade inventiva e de aplicação industrial são protegidos pelo direito de Propriedade Intelectual, via patentes, cujo objetivo é de assegurar aos titulares de invenções a exclusividade na exploração econômica destas, por determinado tempo, seguindo determinados critérios normativos (Zambrano, 2020).

Uma patente é um direito de monopólio temporário concedido a um inventor que lhe permite excluir outros de usar, fazer e vender a invenção protegida, que visa garantir retornos aos investimentos de pesquisa e desenvolvimento desembolsados e promover incentivos à inovação. Devido a pandemia, há uma preocupação de que as patentes, e outras formas de proteção intelectual (PI) possam ser uma barreira na luta contra o COVID-19 (Abi Younes et al., 2020).

Alguns estudiosos apoiam uma abordagem de código aberto, sugerindo dedicar todos as invenções relacionadas ao COVID-19 ao domínio público, ou seja, qualquer pessoa (física ou jurídica) poderão reproduzir e/ou comercializar o produto ou processo decorrente deste conhecimento sem pedir permissão ou pagar royalties ao titular que a inventou, o que torna o acesso a medicamentos mais acessíveis, por exemplo, com surgimento de genéricos. Porém, tal abordagem não reconhece que na busca por vacinas contra o coronavírus, a indústria farmacêutica não unirá forças se nenhuma proteção de patentes ou outra recompensa for fornecida, pois as patentes são concebidas como um valioso incentivo à inovação no ecossistema econômico atual (Van Overwalle, 2020; Zambrano, 2020).

As indústrias farmacêuticas oferecem um argumento para proteção de patentes, argumentando que para criar uma nova droga é arriscado, demorado e muito caro, e uma vez que o composto ativo de uma droga é identificado e testado, fazer uma cópia e produzir a droga geralmente é fácil e a sua produção se torna muito barato. As patentes são vistas como um catalisador para pesquisa e inovação, sendo que o conhecimento é um "bem público", o que significa difícil excluir outras pessoas de usá-lo e que o uso por uma pessoa não reduz sua disponibilidade para outros usuários em potencial (Abi Younes et al., 2020). 
Além das patentes, outros incentivos, como prêmios e subsídios também existem para motivar a inovação tecnológica. Esses incentivos, juntamente com a exigência de que as descobertas resultantes sejam disponibilizadas de forma ampla e aberta, podem alcançar um equilíbrio entre as metas de alocação e inovação (Contreras, 2020).

\subsection{Uso de fármacos contra a Covid-19}

Devido ao cenário crítico imposto pela pandemia, se intensificou a busca por estratégias farmacológicas terapêuticas e/ou profiláticas no combate ao COVID-19. Em diversos países, medicamentos já registrados e utilizados para outras doenças vêm sendo pesquisados como potenciais fármacos para estratégia de segundo uso ("repurposing"), no combate ao coronavírus, como: remdesivir, lopinavir/ritonavir, cloroquina (CQ) e hidroxicloroquina (HQ) (Brito et al., 2020).

A ClinicalTrials.gov, principal base de dados de registros de estudos clínicos dos Estados Unidos, possui mais de 394.619 estudos clínicos registrados em 220 países até 2021 para a Covid-19, considerando-se somente moléculas pequenas e biológicas. Outras importantes bases de dados como a EU Clinical Trials Register, a ISRC-TN e a Chinese Clinical Trial Registry (ChiCTR), também possuem registros que totalizam mais de 2.500 estudos clínicos, com cerca de 200 medicamentos, sem contar fitoterápicos e extratos diversos. A Recovery (Randomised Evaluation of Covid-19) foi estabelecida para investigar a eficácia de diferentes protocolos em pacientes com Covid-19 hospitalizados, sendo liderada pela Universidade de Oxford. Foram considerados os tratamentos com dexametasona (um corticoide com propriedades anti-inflamatórias e imunossupressoras), azitromicina, plasma convalescente, tocilizumabe e REGN-COV2 (Ferreira \& Andricopulo, 2020).

Alguns medicamentos vêm sendo utilizados em vários países nos casos emergenciais da Covid-19 como possível tratamento para a doença, conforme Tabela 1, porém, a maioria desses fármacos não obtiveram eficácia ou ainda estão em estudos prolongados e nenhum deles tem demonstrado ser universalmente eficaz e seguro em grandes triagens clínicas controladas e randomizadas (Brito et al., 2020; de Oliveira, 2020).

Tabela 1 - Fármacos utilizados como possível tratamento para a Covid-19.

\begin{tabular}{cc}
\hline Nome do Fármaco & Utilização \\
\hline Pirfenidona & Utilizado para fibrose pulmonar \\
Ritonavir, Atazanavir, Darunavir, Cobicistat, Realtegravir, & Antirretroviral \\
Tenofovir+emtricitabina, Dolutegravir, Saquinavir, Lamivudina, Tenofovir, & \\
$\begin{array}{c}\text { Lopinavir, Ritonavir, Cobicistrat, Abacavir, Etravirine, Efavirenz, Rilpirivina, } \\
\text { Maraviroc, Bictegravir, Tipranavir, Indinavir, Zidovudina, Remdesivir }\end{array}$ & \\
Ribavirina & Hepatite C crônica e outras \\
& doenças \\
Cloroquina e Hidroxicloroquina & Malária e outras doenças \\
Sarilumabe & Artrite reumatoide \\
Oseltamivir, Arbidol, Umifenovir & Gripe \\
Azitromicina & Antibiótico \\
Aciclovir & Herpes e outras doenças \\
Molnupinavir & Antirretroviral \\
\hline
\end{tabular}

Fonte: Modificado de Silva et al., (2021).

Vários estudos indicaram que não há evidências científicas e benefícios clínicos para o uso da cloroquina e hidroxicloroquina em pacientes com manifestações das formas leve, moderada ou grave do coronavírus. Em razão dos resultados negativos, os fármacos foram descartados dos portifólios de investigação clínica da Recovery e Solidarity (Ferreira \& Andricopulo, 2020).

Recentemente através de pesquisas em fármacos, foi descoberto a eficácia do remédio antirretroviral, o Molnupinavir (Cox et al., 2021) tem sido apontado como uma alternativa terapêutica pra o tratamento da infeção aguda. De acordo com estimativas da empresa farmacêutica Merck Sharp and Dohme (MSD) o medicamento é capaz de reduzir cerca 50\% do risco 
de admissão graves hospitalares à óbitos, mas ressaltam que mesmo com essas condições favoráveis é preciso ter atenção a resistência e o papel das terapias antivirais combinadas (Mahase, 2021).

\subsection{Uso de vacinas contra a Covid-19}

Devido a pandemia do COVID-19, se tornou necessário inovações no desenvolvimento, avaliação e uso de novas vacinas. São 331 vacinas contra COVID-19 estão em desenvolvimento, dentre elas 244 estão na fase pré-clínica e 87 na fase clínica, as quais possuem diferentes plataformas tecnológicas, como vacinas de vetores virais, vacinas baseadas em RNA e DNA recombinante, vacinas de subunidades proteica, partículas semelhantes a vírus (VLP), vírus inativado e vírus atenuada (Brasil, 2020). As vacinas candidatas contra SARS-CoV2 e a plataforma tecnológica são expostas na Tabela 2.

Tabela 2 - Composição das principais vacinas candidatas contra o COVID-19.

\begin{tabular}{ccc}
\hline Plataforma & Tipo de vacina & Vacinas Candidatas \\
\hline PS & Subunidade proteica & 96 \\
VVnr & Vetor Viral (sem replicação) & 62 \\
DNA & DNA & 29 \\
IV & Vírus inativado & 22 \\
RNA & RNA & 41 \\
VLP & Vírus como partícula & 26 \\
LAV & Vírus atenuado vivo & 5 \\
\hline
\end{tabular}

Fonte: Brasil (2020).

Segundo Ritchie et al., (2021) até o momento, 51,5\% da população mundial recebeu pelo menos uma dose de uma vacina COVID-19. Foram administradas 7,41 bilhões de doses globalmente, sendo administradas 28,5 milhões de doses todos os dias. Em países de baixa renda, apenas 4,5\% das pessoas receberam pelo menos uma dose de uma vacina COVID-19.

A eficácia e a segurança são estabelecidas com base na observação entre o produto candidato e um humano em ambiente controlado (Guimarães, 2020). Porém, vacinas eficazes e seguras devem conter critérios que intercedem entre as tecnologias e a sua chegada aos organismos das pessoas que pertençam às populações-alvo a que se destinam. Para ser considerada uma vacina candidata, a vacina deve fornecer uma memória imunológica longa e não deve apresentar manifestações de agravamento da doença, critérios que são relativos à sua efetividade (Guimarães, 2020). Dentre as vacinas candidatas contra SARS-CoV2, 15 estão em avaliação para listagem de uso emergencial segundo a OMS, as quais são representadas na tabela 3. 
Tabela 3 - Vacinas COVID-19 no processo de avaliação para uso emergencial da Organização Mundial de Saúde (OMS).

\begin{tabular}{|c|c|c|c|c|}
\hline $\mathbf{N}^{\mathbf{o}}$ & Fabricantes & Nome da Vacina & $\begin{array}{l}\text { Registro } \\
\text { de RNA }\end{array}$ & Plataforma \\
\hline 1 & Pfizer Biontech & $\begin{array}{l}\text { BNT162b2/COMIRNATY (INN } \\
\text { tozinameran) }\end{array}$ & EMA & mNRA modificado nucleosídeo \\
\hline 2 & $\begin{array}{l}\text { Zhifei Longcom, } \\
\text { China }\end{array}$ & $\begin{array}{l}\text { Recombinante Nova Vacina } \\
\text { Coronavírus (Célula CHO) }\end{array}$ & NMPA & Subunidade de proteína recombinante \\
\hline 3 & IMBCAMS, China & $\begin{array}{c}\text { Vacina SARS-CoV-2, Inativada } \\
\text { (célula verdadeira) }\end{array}$ & NMPA & Inativada \\
\hline 4 & AstraZeneca Oxford & AZD1222 & $\begin{array}{l}\text { Core- EMA } \\
\text { Non } \\
\text { COVAX }\end{array}$ & $\begin{array}{c}\text { Recombinação de adenovírus de } \\
\text { chimpanzé defeituoso expressando a } \\
\text { glicoproteína superficial SARS-CoV-2 } \\
\text { S }\end{array}$ \\
\hline 5 & SKBIO & AZD1222 & $\begin{array}{c}\text { MFDS } \\
\text { KOREA }\end{array}$ & - \\
\hline 6 & Janssen & Ad26.COV2.S & EMA & $\begin{array}{l}\text { Vacina vetorial recombinante, } \\
\text { replicação incompetente tipo } 26 \\
\text { (Ad26) codificação da proteína } \\
\text { (SARS-CoV-2) Spike (S) }\end{array}$ \\
\hline 7 & Sinopharm & $\begin{array}{c}\text { Vacina SARS-CoV-2 (Célula } \\
\text { verdadeira), Inativada (INCOV) }\end{array}$ & NMPA & Inativado, produzido em células Vero \\
\hline 8 & Sinovac & $\begin{array}{c}\text { Vacina SARS-CoV-2 (Células } \\
\text { verdadeiras), Inativada }\end{array}$ & NMPA & $\begin{array}{c}\text { Inativado, produzido em células } \\
\text { verdadeiras }\end{array}$ \\
\hline 9 & $\begin{array}{l}\text { The Gamaleya } \\
\text { National Center }\end{array}$ & Sputnik V & $\begin{array}{c}\text { Russian } \\
\text { NRA }\end{array}$ & $\begin{array}{c}\text { Uma vacina Covid-19 baseada em } \\
\text { vetores de Adenovírus }\end{array}$ \\
\hline 10 & $\begin{array}{l}\text { Centro Estadual de } \\
\text { Pesquisa vetorial de } \\
\text { Virologia e } \\
\text { Biotecnologia }\end{array}$ & EpiVacCorona & $\begin{array}{c}\text { Russian } \\
\text { NRA }\end{array}$ & Antígeno de peptídeos \\
\hline 11 & CanSinoBio & Ad5-nCoV & & $\begin{array}{c}\text { Recombinação Nova Vacina } \\
\text { Coronavírus (Vetor Adenovírus Tipo } \\
5)\end{array}$ \\
\hline 12 & Moderna & mRNA-1273 & EMA & $\begin{array}{c}\text { vacina à base de mNRA encapsulada } \\
\text { em nanopartícula lipídica (LNP) }\end{array}$ \\
\hline 13 & Instituto Soro da Índia & $\begin{array}{c}\text { Covishield (ChAdOx1_nCoV- } \\
19)\end{array}$ & DCGI & $\begin{array}{l}\text { Vetor adenoviral ChAdOx1 } \\
\text { recombinante codificando o antígeno } \\
\text { de proteína spike do SARS-CoV-2 }\end{array}$ \\
\hline 14 & Sinopharm & - & NMPA & $\begin{array}{l}\text { Nenhuma reunião de pré-submissão } \\
\text { ainda }\end{array}$ \\
\hline 15 & Novavax & - & EMA & $\begin{array}{c}\text { Nenhuma reunião de pré-submissão } \\
\text { ainda }\end{array}$ \\
\hline
\end{tabular}

Fonte: Modificado de Organização Mundial de Saúde (2021a).

\section{Covid-19 no Brasil}

\subsection{Histórico e evolução do covid-19 no Brasil}

O primeiro caso confirmado no Brasil ocorreu em 26 de fevereiro de 2020, um homem de 61 anos que viajou pela Itália e chegou a apresentar os sintomas da doença (paciente zero). Dois dias após, 182 casos suspeitos foram registrados da doença em 16 estados do Brasil. Em 30 de Janeiro, a Organização Mundial de Saúde decretou Emergência em Saúde Pública de Importância Internacional, e em 3 de fevereiro de 2020 o Ministério da Saúde decretou Emergência em Saúde Pública de importância Nacional (ESPIN) em decorrência da Infecção Humana pelo novo Coronavírus (2019-nCoV) segundo a portaria ${ }^{\circ}$ 188. 
O primeiro óbito registrado em território brasileiro ocorreu em 17 de março, e atualmente no Brasil há 19.688.663 de casos acumulados e 549.924 óbitos (CONASS, 2021). O Brasil possui uma população estimada em 213.384.768 de habitantes (IBGE, 2021), é o segundo país com maior número de casos, e recentemente atingiu a margem de maior número de mortes diárias, 4.249 vidas perdidas foram registradas em 8 de abril de 2021 (Brasil, 2021). A falta de implementação da testagem em massa da população pode aumentar o número de casos e óbitos por subnotificação (Aquino et al., 2020), no entanto, espera-se que esse número possa ser revertido com a implementação das campanhas de vacinação.

Atualmente, o Brasil está entre os países que aprovaram o uso emergencial de 4 vacinas candidatas contra SARSCoV2, são elas: Coronavac, Covisheield, Comirnaty e Janssen (Brasil, 2021). A vacina Coronavac está sendo desenvolvida pela empresa biofarmacêutica chinesa Sinovac Biotech e produzida no Brasil com o apoio tecnológico do Instituto Butantan, em São Paulo. CoronaVac é uma vacina inativada contra COVID-19, criada a partir de células de rim de macaco verde africano (células Vero) que foram inoculadas com SARS-CoV-2 (cepa CN02), sua eficácia é de 50,38\% na prevenção contra COVID-19, após a aplicação de duas doses, importante ressaltar que a Coronavac mostrou-se $100 \%$ eficaz nos casos moderados e graves e 78\% nos casos leves da COVID-19 (Zhang et al., 2021).

A vacina Covishiled, conhecida também como vacina de Oxford/AstraZeneca, está sendo desenvolvida pela empresa AstraZeneca e produzida no Brasil em parceria com a Fundação Oswaldo Cruz (Fiocruz). A vacina Covishiled é uma vacina de vetor de adenovírus de chimpanzé modificado que codifica a proteína de pico do coronavírus da síndrome respiratória do Oriente Médio (MERS-CoV), sua eficácia é de 70\% após a aplicação de suas doses (Folegatti et al., 2020).

Já a vacina Cominarty conhecida como Pfizer é uma vacina de RNA modificada produzida pela Pfizer com parceria do laboratório BioNTech, sua eficácia é de 95\% após a aplicação de duas doses (Polack et al., 2020). E recentemente foi aprovada a vacina Janssen, um vetor adenovírus humano, que está sendo produzida pela Johnson \& Johson, sua eficácia é de 66,9\% após a aplicação de uma dose (Sadoff et al., 2021).

\subsection{Ciência brasileira frente à pandemia}

Em decorrência da pandemia causada pelo coronavírus, é enfatizada a relevância da contribuição científica dos institutos de educação e pesquisa à sociedade como um todo. Para enfrentar o COVID-19, o Estado e a sociedade buscam apoio da ciência e das instituições de pesquisa para combatê-la. No Brasil, os polos de pesquisa estão concentrados nas instituições federais de ensino superior (IFES), especialmente nas universidades públicas. Essas instituições possuem infraestrutura física e humana para implementar, rapidamente, ações que integram a pesquisa e a extensão de problemas complexos e com impacto em diferentes áreas, como Saúde, Economia, Ambiente e Sociedade.

As ações desenvolvidas pelas universidades públicas podem gerar reflexos e contribuições efetivas (Almeida et al., 2020; Panizzon et al., 2020). De acordo com o ministério da saúde, atualizado em 9 de abril de 2021, são 18 vacinas candidatas contra SARS-CoV2 desenvolvimento por diferentes institutos brasileiros listadas na tabela 4. A qual, somente a vacina de vírus inativado, utilizando o vírus da doença de Newcastle desenvolvida pelo Instituto Butantan se encontra na fase clínica I/II, as demais vacinas candidatas se encontram na fase pré-clínica. 
Tabela 4 - Instituições de desenvolvimento de vacinas brasileiras e a tecnologia/tipo de vacina utilizado.

\begin{tabular}{|c|c|}
\hline Instituição de desenvolvimento & Tecnologia/Tipo de vacina \\
\hline Instituto Butantan & $\begin{array}{c}\text { Vacina baseada em partículas semelhantes ao } \\
\text { vírus (VLP-Virus-Like-Particle) }\end{array}$ \\
\hline Instituto Butantan & $\begin{array}{c}\text { Vacina de vírus inativado da Doença de } \\
\text { Newcastle (NDV)- inativado que expressa a } \\
\text { proteína SARS-CoV }\end{array}$ \\
\hline Instituto Butantan & $\begin{array}{l}\text { Vesículas de membrana externa em plataforma } \\
\text { de múltiplos antígenos }\end{array}$ \\
\hline $\begin{array}{c}\text { Instituto do Coração (Incor) da Faculdade de } \\
\text { Medicina da USP (FMUSP)/ Universidade de São } \\
\text { Paulo (USP) }\end{array}$ & $\begin{array}{c}\text { Vacina baseada em partículas semelhantes ao } \\
\text { vírus (VLP-Vírus-Like-Particle) }\end{array}$ \\
\hline $\begin{array}{l}\text { Instituto de Ciências Biomédicas da Universidade } \\
\text { de São Paulo (USP) }\end{array}$ & Vacina de ácido nucleico (DNA) \\
\hline $\begin{array}{l}\text { Instituto de Ciências Biomédicas da Universidade } \\
\text { de São Paulo (USP) }\end{array}$ & Vacina baseada em nanopartículas \\
\hline $\begin{array}{l}\text { Instituto de Ciências Biomédicas da Universidade } \\
\text { de São Paulo (USP) }\end{array}$ & Vacinas baseadas em proteína recombinante \\
\hline Universidade Federal de Viçosa & Vacinas baseadas em proteína recombinante \\
\hline $\begin{array}{c}\text { Faculdade de Ciências Farmacêuticas da } \\
\text { Universidade de São Paulo (USP) }\end{array}$ & Vacina baseada em nanopartículas \\
\hline Universidade Federal do Paraná (UFPR) & Vacina baseada em nanopartículas \\
\hline Faculdade de Zootecnia e Engenharia de & Vacina baseada em vetores virais \\
\hline Alimentos, Universidade de São Paulo (USP) & \\
\hline Universidade Federal de Minas Gerais (UFMG) & Vacina de ácido nucleico (DNA) \\
\hline $\begin{array}{c}\text { Empresa Farmacore em parceria com PDS Biotech } \\
\text { e Faculdade de Medicina de Ribeirão Preto } \\
\text { (FMRP-USP) }\end{array}$ & $\begin{array}{c}\text { Vacina Versamune-CoV-2F combina uma } \\
\text { proteína recombinante com a nanotecnologia da } \\
\text { plataforma Versamune }\end{array}$ \\
\hline Bio-Manguinhos/Fiocruz & Vacina sintética \\
\hline Bio-Manguinhos/Fiocruz & Vacina baseada em subunidade proteica \\
\hline Instituto René Rachou (Fiocruz/MG)/Instituto & Vacina baseada em vetores virais \\
\hline $\begin{array}{c}\text { Nacional de Ciência e Tecnologia em Vacinas } \\
\text { (INCTV) }\end{array}$ & \\
\hline Universidade Federal do Paraná (UFPR) & Vacina baseada em nanopartículas \\
\hline Universidade Federal do Rio de Janeiro (UFRJ) & Ácido Nucleico \\
\hline Universidade Federal de Minas Gerais (UFMG) & Quimera proteica \\
\hline
\end{tabular}

Fonte: Brasil (2020).

\subsection{Desafios de efetuar medidas preventivas ao covid-19 no Brasil}

No início da pandemia no Brasil, o Ministério da Saúde (Brasil, 2020c) dispôs algumas medidas de prevenção para conter o vírus, entre elas destacam-se: lavar as mãos com água e sabão ou álcool em gel 70\%, manter a distância de 1 (um) metro entre pessoas nos locais públicos, não compartilhamento de objetos sociais e uso de máscara em todos os ambientes. Além disso, também foi restringido o desembarque de estrangeiros em porto em todo o território brasileiro de 26 de março de 2020 a 26 de abril de 2020 (Brasil, 2020c).

É válido ressaltar que países com dimensões continentais como o Brasil, com uma população extensa e um país com grande desigualdade social, há populações que vivem em condições precárias, como falta de saneamento, falta de recursos dos serviços de saúde e acesso limitado à educação de qualidade (Werneck \& Carvalho, 2020). Nesse sentido, as políticas públicas são de extrema importância para garantir os direitos básicos, como saneamento, água de qualidade, garantia de recursos à saúde para todos, garantindo assim que as medidas de prevenção ao COVID-19 sejam eficientes (Macedo et al., 2020). Além disso, nessas regiões os recursos de atenção à saúde são insuficientes, portanto, a adoção de medidas mais rigorosas de distanciamento social será um determinante para reduzir o colapso dos serviços de saúde (Aquino et al., 2020). 
Estudos recentes mostraram o impacto do isolamento social no combate à pandemia causada pelo COVID-19. Pesquisas apontam que o crescimento do isolamento social está associado negativamente com a velocidade dos casos de COVID-19 (Silva et al., 2021). Também relatam que a medida de bloqueio, lockdown em inglês, é uma medida efetiva para minimizar o impacto, atuando na redução de casos confirmados (Houvèssou et al., 2021). Os autores ainda relatam que o crescente aumento no número de casos no Brasil e nos Estados Unidos, podem ser devido à falta de políticas de lockdown. Estima-se que até centenas de milhares das mortes poderiam ter sido evitadas no Brasil devido ao conjunto de ações equivocadas e omissões do poder público (Hallal, 2021).

Segundo um modelo de taxa de letalidade bayesiana utilizado por Brizzi e colaboradores (2021) foi observado que as flutuações geográficas e temporais nas taxas de letalidade hospitalar do COVID-19 no Brasil estão principalmente associadas a iniquidades geográficas e escassez de capacidade de atendimento à saúde. Foi possível observar no período pandêmico a grande desigualdade de recursos de saúde em todo o país. Ainda de acordo com os autores, metade das mortes por COVID - 19 no Brasil em hospitais poderiam ter sido evitadas sem desigualdades geográficas pré-pandêmicas e sem pressão de saúde pandêmica. Os óbitos hospitalares poderiam ter sido reduzidos em cerca de 56,55\% (53,95\% -59,22\%) nas 14 capitais do Brasil (Brizzi et al., 2021).

As vacinas também são uma importante ferramenta na contenção do vírus, e devem estar alinhadas em conjunto com outras medidas de saúde pública (Kim et al., 2021). Nesse contexto, é importante ressaltar a necessidade da alfabetização em saúde da sociedade, considerando a grande quantidade de notícias contraditórias sobre a eficácia das vacinas contra SARSCoV2 (Biasio et al., 2021). Recentemente pesquisadores avaliaram o conhecimento de COVID-19 no Brasil e concluiu que os participantes com maior escolaridade estavam mais bem informados sobre a doença. Os autores ainda ressaltam a importância do investimento na educação, pois além de contribuir para o conhecimento científico, criam uma população mais informada, reduzindo assim o compartilhamento de fake news, e aumentando a disseminação de informações científicas sobre a importância da vacinação e das medidas preventivas de contenção do vírus (Guimarães et al., 2021).

Até o presente momento (10/11/2021) cerca de 75,29\% da população brasileira foram vacinadas, dentre esses, 58,25\% totalmente imunizadas, e 17,04\% parcialmente (Ritchie et al., 2021). Com o progresso da vacinação podemos observar claramente uma redução da curva de casos e óbitos decorrente da Covid-19. Comparando dados de abril de 2021, a taxa de vacinação diária era cerca de 940 mil, e a taxa diária de óbitos era de 14,49 por milhão de pessoas. Com o avanço da administração de doses diárias em agosto de 2021 com 2,05 milhões doses diárias, a taxa de óbitos diários passa de 14,49 para 3,89 óbitos por milhão de pessoas, e recentemente esse número caiu para 1,41 em 01 de novembro de 2021 (Ritchie et al., 2021). Nesse contexto se faz importante ressaltar a centralidade do SUS no sucesso na vacinação e no combate à pandemia. De acordo com (Silva e Ruiz., 2020) é necessário defender o SUS como modelo de saúde, pois diante o cenário de pandemia onde o quadro de morte de pessoas é crescente, não é possível naturalizar sistemas de saúde que diferenciam leitos públicos e privados, além disso destacam a inacessibilidade financeira dos planos de saúde. Em comparação com outros países, mesmo o Brasil tendo a vacinação atrasada por políticas públicas, o SUS conseguiu que em menos de um ano grande parte da população fosse vacinada em um curto espaço de tempo.

\section{Considerações Finais}

A pandemia causada pelo coronavírus é a segunda do século e a maior em termos de transmissão e taxa de mortalidade. A presença da vida humana cada vez maior em ambientes silvestres com a degradação ambiental e venda ilegal de animais, faz com que epidemias e pandemias sejam cada vez mais susceptíveis de se ocorrer. Devido a crítica situação imposta pela pandemia, cientistas do mundo inteiro intensificaram a busca por soluções, dentre elas estratégicas farmacológicas e terapêuticas ao tratamento de COVID-19. 
O uso de medicamentos no início de 2020 foi bastante explorado, mas nem todos tiveram estudos suficientes e verídicos da sua eficácia, e acabou gerando uma má desinformação e uso inadequado, como o caso da cloroquina (CQ) e hidroxicloroquina (HQ). Mas, com a capacidade dos cientistas e pesquisadores, em 2021 foi possível um fato inédito com a produção da vacina em um tempo recorde, e também a descoberta de um medicamento terapêutico capaz de tratar a infecção aguda e reduzir cerca de $50 \%$ dos óbitos, com o nome de Monulpinavir. Com tudo isso, mesmo com grandes esforços, países subdesenvolvidos e grandes em termos de expansão territorial, ainda sofre com a lentidão e a falta de interesse público em minimizar a taxa de transmissão e aumentar a taxa de vacinação, como é o caso do Brasil. O SUS é um dos maiores sistemas públicos de saúde do mundo, diante disso se faz importante a valorização dos profissionais da saúde e pesquisadores no Brasil, que tiveram um trabalho árduo para conter e minimizar a pandemia. Atualmente, até o fechamento deste artigo, o Brasil se encontra com cerca de $56,06 \%$ da população totalmente imunizada com duas doses, e são cerca de 276.427 .527 doses aplicadas dentre as vacinas disponíveis, é um grande avanço que só foi possível devido ao desenvolvimento da vacina e uma população consciente, da importância da vacinação.

O Brasil está em $4^{\circ}$ lugar em relação aos outros países quanto à imunização, sendo 160,11 milhões de pessoas que receberam pelo menos uma dose de vacina e 121,13 milhões de pessoas que foram completamente vacinadas, e esse número cresce a cada dia. Isso se deve a baixa taxa de hesitação vacinal entre os brasileiros. Portanto, nosso trabalho reforça as necessidades de medidas de contenção do vírus, a necessidade do isolamento social para diminuir a velocidade de transmissão do vírus, e do investimento na saúde e educação para a redução da disseminação de informações falsas e para a compreensão da importância da vacinação, além disso, ressalta a relevância de políticas públicas eficazes e de direito a todos.

Esse artigo auxilia trabalhos presentes sobre a atual situação da pandemia, como o vírus se conduziu no Brasil e os avanços das tecnologias. Para possíveis estudos futuros, sugere-se a atualização dos dados referente as vacinas, demonstrando como as medidas e esforço da ciência são necessários, e relacioná-los com a evolução dos estudos do coronavírus no Brasil.

Dedicado à memória de Stênio Nunes Alves, pró-reitor de Pesquisa e Pós-Graduação da UFSJ.

\section{Referências}

Abi Younes, G., Ayoubi, C., Ballester, O., Cristelli, G., de Rassenfosse, G., Foray, D., Gaulé, P., Pellegrino, G., van den Heuvel, M., Webster, E., \& Zhou, L. (2020). COVID-19: Insights from innovation economists. Science and Public Policy, 1-13. https://doi.org/10.1093/scipol/scaa028

Almeida, L. de S. B., Silveira, R. M. da C., Silva, B. C. do N., Queiroz, J. V. R. de, \& Oliveira, P. H. C. do N. de. (2020). As universidades públicas brasileiras no contexto da pandemia: iniciativas e parcerias no enfrentamento da covid-19. Cadernos Gestão Pública e Cidadania, 25(82), 1-20. http://dx.doi.org/10.12660/cgpc.v25n82.82123

Aquino, E. M. L., Silveira, I. H., Pescarini, J. M., Aquino, R., \& de Souza-Filho, J. A. (2020). Social distancing measures to control the COVID-19 pandemic: Potential impacts and challenges in Brazil. Ciencia e Saude Coletiva, 25, 2423-2446. https://doi.org/10.1590/1413-81232020256.1.10502020

Biasio, L. R., Bonaccorsi, G., Lorini, C., \& Pecorelli, S. (2021). Assessing COVID-19 vaccine literacy: a preliminary online survey. Human Vaccines and Immunotherapeutics, 17(5), 1304-1312. https://doi.org/10.1080/21645515.2020.1829315

Bliddal, S., Banasik, K., Pedersen, O. B., Nissen, J., Cantwell, L., Schwinn, M., Tulstrup, M., Westergaard, D., Ullum, H., Brunak, S., Tommerup, N., Feenstra, B., Geller, F., Ostrowski, S. R., Grønbæk, K., Nielsen, C. H., Nielsen, S. D., \& Feldt-Rasmussen, U. (2021). Acute and persistent symptoms in nonhospitalized PCR-confirmed COVID-19 patients. Scientific Reports, 11(1), 1-11. https://doi.org/10.1038/s41598-021-92045-X

Blomberg, B., Mohn, K. G.-I., Brokstad, K. A., Zhou, F., Linchausen, D. W., Hansen, B.-A., Lartey, S., Onyango, T. B., Kuwelker, K., Sævik, M., Bartsch, H., Tøndel, C., Kittang, B. R., Madsen, A., Bredholt, G., Vahokoski, J., Fjelltveit, E. B., Bansal, A., Trieu, M. C., \& Langeland, N. (2021). Long COVID in a prospective cohort of home-isolated patients. Nature Medicine. https://doi.org/10.1038/s41591-021-01433-3

Bonadio, E., \& Baldini, A. (2020). Covid-19, patents and the never-ending tension between proprietary rights and the protection of public health. European Journal of Risk Regulation, 11(2), 390-395. https://doi.org/10.1017/err.2020.24

Brasil. (2020). Relatório Técnico - Monitoramento de vacinas em desenvolvimento contra Sars-CoV-2. 30 de novembro de 2020. Ministério da Saúde. Secretaria de Ciência, Tecnologia, Inovação e Insumos Estratégicos Em Saúde. Departamento de Ciência e Tecnologia.

Brasil. (2020a). PORTARIA No 1.565 , DE 18 DE JUNHO DE 2020. Estabelece orientações gerais visando à prevenção, ao controle e à mitigação da transmissão da COVID-19, e à promoção da saúde física e mental da população brasileira, de forma a contribuir com as ações para a re. Diário Oficial Da 
União. Brasilia. http://www.in.gov.br/en/web/dou/-/portaria-n-1.565-de-18-de-junho-de-2020-262408151

Brasil. (2020b). Portaria No 47, de 26 de março de 2020. Dispõe sobre a restrição excepcional e temporária de entrada no País de estrangeiros por transporte aquaviário, conforme recomendação da Agência Nacional de Vigilância Sanitária - Anvisa. Ministério da Saúde. Diário Oficial Da União. Brasilia.

Brito, J. C. M., Lima, W. G., Cardoso, B. G., Simião, D. C., Amorim, J. M., \& Silva, C. de A. (2020). Uso irracional de medicamentos e plantas medicinais contra a COVID-19 (SARS-CoV-2): Um problema emergente. Brazilian Journal of Health and Pharmacy, 2(3), 37-53. https://doi.org/10.29327/226760.2.3-5

Brizzi, A., Whittaker, C., Servo, L., Hawryluk, I., Prete, C. A., de Souza, W. M., Aguiar, R. S., Araujo, L., Bastos, L. S., Blenkinsop, A., Buss, L. F., Candido, D., Castro, M. C., Costa, S. F., Croda, J., de Souza Santos, A. A., Dye, C., Flaxman, S., Fonseca, P., Geddes, V., ... Ratmann, O. (2021). Report 46: Factors driving extensive spatial and temporal fluctuations in COVID-19 fatality rates in Brazilian hospitals. medRxiv : the preprint server for health sciences, 2021.11.01.21265731. https://doi.org/10.1101/2021.11.01.21265731

CONASS (2021). Conselho Nacional de Secretários de Saúde. Painel CONASS COVID-19. https://www.conass.org.br/painelconasscovid19/.

Contreras, J. L. (2020). Expandindo o acesso a patentes para COVID-19. 50.

Cox, R. M., Wolf, J. D., \& Plemper, R. K. (2021). Therapeutically administered ribonucleoside analogue MK-4482/EIDD-2801 blocks SARS-CoV-2 transmission in ferrets. Nature Microbiology, 6(1), 11-18. https://doi.org/10.1038/s41564-020-00835-2

de Oliveira, E. H. A. (2020). Coronavírus: prospecção científica e tecnlógica dos fármacos em estudo para tratamento da Covid-10. Cadernos de Prospecção, 13(2), 412-423. http://dx.doi.org/10.9771/cp.v13i2.COVID-19.36153

Ferreira, L. L. G., \& Andricopulo, A. D. (2020). Medicamentos e tratamentos para a Covid-19. Estudos Avancados, 34(100), 7-27. https://doi.org/10.1590/s0103-4014.2020.34100.002

Folegatti, P. M., Ewer, K. J., Aley, P. K., Angus, B., Becker, S., Belij-rammerstorfer, S., Bellamy, D., Bibi, S., Bittaye, M., Clutterbuck, E. A., Dold, C., Faust, S. N., Finn, A., Flaxman, A. L., Hallis, B., Heath, P., Jenkin, D., Lazarus, R., Makinson, R., ... Pollard, A. J. (n.d.). Articles Safety and immunogenicity of the ChAdOx1 nCoV-19 vaccine against SARS-CoV-2: a preliminary report of a phase 1/2, single-blind, randomised controlled trial. $467-478$.

Funk, C. D., Laferrière, C., \& Ardakani, A. (2020). A Snapshot of the Global Race for Vaccines Targeting SARS-CoV-2 and the COVID-19 Pandemic. Frontiers in Pharmacology, 11(June), 1-17. https://doi.org/10.3389/fphar.2020.00937

Guimarães, R. (2020). Anti-covid vaccines: A look from the collective health. Ciencia e Saude Coletiva, 25(9), 3579-3585. https://doi.org/10.1590/141381232020259.24542020

Guimarães, V. H. A., de Oliveira-Leandro, M., Cassiano, C., Marques, A. L. P., Motta, C., Freitas-Silva, A. L., de Sousa, M. A. D., Silveira, L. A. M., Pardi, T. C., Gazotto, F. C., Silva, M. V., Rodrigues, V., Rodrigues, W. F., \& Oliveira, C. J. F. (2021). Knowledge about COVID-19 in Brazil: Cross-sectional webbased study. JMIR Public Health and Surveillance, 7(1). https://doi.org/10.2196/24756

Hallal, P. C. (2021). SOS Brazil: science under attack. The Lancet, 397(10272), 373-374. https://doi.org/10.1016/S0140-6736(21)00141-0

Houvèssou, G. M., Souza, T. P. de, \& Silveira, M. F. da. (2021). Medidas de contenção de tipo lockdown para prevenção e controle da COVID-19: estudo ecológico descritivo, com dados da África do Sul, Alemanha, Brasil, Espanha, Estados Unidos, Itália e Nova Zelândia, fevereiro a agosto de 2020. Epidemiologia e Servicos de Saude : Revista Do Sistema Unico de Saude Do Brasil, 30(1), e2020513. https://doi.org/10.1590/S1679-49742021000100025

Instituto Brasileiro de Geografia e Estatística - IBGE (2021). População do Brasil. https://www.ibge.gov.br/apps/populacao/projecao/box_popclock.php.

Kim, J. H., Marks, F., \& Clemens, J. D. (2021). Looking beyond COVID-19 vaccine phase 3 trials. Nature Medicine, 27(2), 205-211. https://doi.org/10.1038/s41591-021-01230-y

Lima, E. J. da F., Almeida, A. M., \& Ávila, R. de. (2021). Vacinas para COVID-19 - o estado da arte. Rev. Bras. Saúde Matern. Infant, 21 , 21-27. http://dx.doi.org/10.1590/1806-9304202100S100002

Macedo, Y. M., Ornellas, J. L., \& Bomfim, H. F. do. (2020). COVID - 19 NO BRASIL: o que se espera para população subalternizada? Revista Encantar, 110. https://doi.org/10.5935/encantar.v2.0001

Mahase, E. (2021). Covid-19: Molnupiravir reduces risk of hospital admission or death by 50\% in patients at risk, MSD reports. Bmj, October, n2422. https://doi.org/10.1136/bmj.n2422

Ministério da saúde (MS). COVID-19 Painel Coronavírus. Brasília: MS; 2021. Disponível em: https://covid.saude.gov.br/ https://covid.saude.gov.br/. (2021). 2021.

Moreira, R. M., Villa Montoya, A. C., Silveria Araujo, S. L., Trindade, R. A., da Cunha Oliveira, D., \& de Oliveira Marinho, G. (2020). How prepared is Brazil to tackle the COVID-19 disease? Journal of Global Health, 10(2), 020321. https://doi.org/10.7189/jogh.10.020321

Nalbandian, A., Sehgal, K., Gupta, A., Madhavan, M. V., McGroder, C., Stevens, J. S., Cook, J. R., Nordvig, A. S., Shalev, D., Sehrawat, T. S., Ahluwalia, N., Bikdeli, B., Dietz, D., Der-Nigoghossian, C., Liyanage-Don, N., Rosner, G. F., Bernstein, E. J., Mohan, S., Beckley, A. A., \& Wan, E. Y. (2021). Post-acute COVID-19 syndrome. Nature Medicine, 27(4), 601-615. https://doi.org/10.1038/s41591-021-01283-z

Panizzon, M., Costa, C. F. da, \& Medeiros, I. B. de O. (2020). Práticas das universidades federais no combate à COVID-19: a relação entre investimento público e capacidade de implementação. Revista de Administração Pública, 54(4), 635-649. https://doi.org/10.1590/0034-761220200378

Polack, F. P., Thomas, S. J., Kitchin, N., Absalon, J., Gurtman, A., Lockhart, S., Perez, J. L., Pérez Marc, G., Moreira, E. D., Zerbini, C., Bailey, R., Swanson, K. A., Roychoudhury, S., Koury, K., Li, P., Kalina, W. V., Cooper, D., Frenck, R. W., Hammitt, L. L., \& Gruber, W. C. (2020). Safety and Efficacy of the BNT162b2 mRNA Covid-19 Vaccine. New England Journal of Medicine, 383(27), 2603-2615. https://doi.org/10.1056/nejmoa2034577 
Research, Society and Development, v. 10, n. 16, e235101623493, 2021

(CC BY 4.0) | ISSN 2525-3409 | DOI: http://dx.doi.org/10.33448/rsd-v10i16.23493

Ritchie, H., Mathien, E., Rodés-Guirao, L., Appel, C., Giattino, C., Ortiz-Ospina, E., Hasell, J., Macdonald, B., Beltekian, D., \& Roser, M. (2020) "Coronavirus Pandemic (COVID-19)".http://ourworldindata.org/coronavirus'

Sadoff, J., Gray, G., Vandebosch, A., Cárdenas, V., Shukarev, G., Grinsztejn, B., Goepfert, P. A., Truyers, C., Fennema, H., Spiessens, B., Offergeld, K., Scheper, G., Taylor, K. L., Robb, M. L., Treanor, J., Barouch, D. H., Stoddard, J., Ryser, M. F., Marovich, M. A., \& Douoguih, M. (2021). Safety and Efficacy of Single-Dose Ad26.COV2.S Vaccine against Covid-19. New England Journal of Medicine, 384(23), 2187-2201. https://doi.org/10.1056/nejmoa2101544

Silva, F. C. da, Zamprogna, K. M., Souza, S. S. de, \& Sell, D. H. S. D. (2021). Isolamento social e a velocidade de casos de covid-19: medida de prevenção da transmissão. Revista Gaucha de Enfermagem, 42, e20200238. https://www.seer.ufrgs.br/RevistaGauchadeEnfermagem/article/view/110513

Silva, K. M. R. da, Silva, P. B. da, Silva, H. J. N. da, Silva, P. T. da, Silva, B. L., Ferreira, A. M. C., Bezerra, W. A. dos S., Silva, D. de A. e, Campos, M. R. de, Oliveira, D. M. T. e, Nascimento, A. L. L. do, Ribeiro, A. M. de C., Silva, A. M. da, Sousa, E. A., Oliveira, I. K. F., Lopes, M. M. F., Andrade, N. B. de, Pinheiro, I. M., Ferreira, K. L. C., \& Furtado, D. R. L. (2021). Avaliação dos principais fármacos utilizados para o sars-cov-2: uma prospecção cientifica e tecnológica. Research, Society and Development, 2021, 1-14. https://doi.org/10.33448/rsd-v10i3.13136

Van Overwalle, G. (2020). Will Covid Patents Save the World? GRUR International, 69(9), 883-884. https://doi.org/10.1093/grurint/ikaa110 Welison Matheus Fontes da Silva e Jefferson Lee de Souza Ruiz. (2020). Coronavírus E As Disputas Com O Projeto Neoliberal. 30(3), 1-8.

Werneck, G. L., \& Carvalho, M. S. (2020). A pandemia de COVID-19 no Brasil: Crônica de uma crise sanitária anunciada. Cadernos de Saude Publica, 36(5), 1-4. https://doi.org/10.1590/0102-311X00068820

World Health Organization (2021a). COVID-19 vaccine tracker and landscape: Status of COVID-19 Vaccines within WHO EUL/PQ evaluation process. https://www.who.int/publications/m/item/draft-landscape-of-covid-19-candidate-vaccines.

World Health Organization (2021). Painel do Coronavírus da OMS (COVID-19) https://covid19.who.int/.

Zambrano, V. (2020). O Direito À Saúde E À Vida Em Confronto Com O Direito À Propriedade Intelectual Dos Laboratórios , No Âmbito Da Pandemia Da Covid 19: a Possivel Quebra De Patentes. 05, 168-192.

Zhang, J. jin, Dong, X., Cao, Y. yuan, Yuan, Y. dong, Yang, Y. bin, Yan, Y. qin, Akdis, C. A., \& Gao, Y. dong. (2020). Clinical characteristics of 140 patients infected with SARS-CoV-2 in Wuhan, China. Allergy: European Journal of Allergy and Clinical Immunology, 75(7), 1730-1741. https://doi.org/10.1111/all.14238

Zhang, Y., Zeng, G., Pan, H., Li, C., Hu, Y., Chu, K., Han, W., Chen, Z., Tang, R., Yin, W., Chen, X., Hu, Y., Liu, X., Jiang, C., Li, J., Yang, M., Song, Y., Wang, X., Gao, Q., \& Zhu, F. (2021). Safety, tolerability, and immunogenicity of an inactivated SARS-CoV-2 vaccine in healthy adults aged 18-59 years: a randomised, double-blind, placebo-controlled, phase 1/2 clinical trial. The Lancet Infectious Diseases, 21(2), 181-192. https://doi.org/10.1016/S14733099(20)30843-4 